i. J. Batto

R. H. Pratt

Nay 1979

P.0. 9776403

UNIVLRSITY OF PITISBURGH

PIIIISBURCII, PENSSYLYNIA 


\title{
Compton Scattcring̨:
}

\author{
David J. Botto and R. "l. Prate \\ Department of Physics and istronomy \\ University of pittaburgh \\ Pittsburgh, Pennsylvania 15260
}

\begin{abstract}
The current status of Compton scattering, hoth oxperincrtal observations and the theoretical predictions, is cxininec. Classes of experitents are distinguished and the results abtained are suanerized. 'Lie validity of the incoherent scatcorin;, function approximation and the inpulse approxilation is discussed. These simple theoretical approaches are compared with prodictions of the nonrelativistic dipole foraula of Gavrlla and with the relativistic results of thiteinghas. It is noted that the $\bar{A}^{2}$ hatsed
\end{abstract}

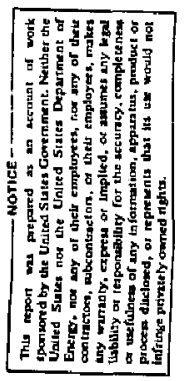


spproximations fall to predict resonances and an infrared divergence, both of which have been observed. It appears that at present the vartous avallable theoretical approaches dLfer slgnificancly in their predictions and that further and nore systematic work is requited. 
Table of Contents

I Introduction

II Scattering from free electrons

III Compton scattering from bound electrons

A. Nonrelativistic Formalism

B. Hydrogenlike Case

C. The $A^{2}$ Approximation

D. Incoherent Scattering Factor

E. Inpulse Approximation

F. Relativistic Approaches

C. Comparison of theoretical approaches

IV Experiments and comparison to theoretical work

A. lleasurenents of $J(q)$

B. lleasurements of $d^{2} \sigma / d ? \mathrm{~d}_{2}$

C. Heasurenents of $\mathrm{do} / \mathrm{d}$ ?

$v$ Low energy Compton scattering and the Resonant Ranan effect

vI Conclusions 
I. Introduction

Compton scattering $[1,2]$ is the incoherent (inelastic) scattering of a photon by an electron: which then recolls. Thus, if the electron is infefally: bound. the Compton process is linited to the struation in which the atom is ionized (Compton scattering), rather than excited. (Raman scattering) or: left in its initial state (Rayleigh scattering).. Here we- restrict:our attention to the situation in which one photon.is.scattered, not considering multiphoton or many electron effects or.processes. As in all quantum electrodynamic processes, there are higher order radiative corrections to the basic calculatior we are describing. The magnitude of such effects depends on the-sensitivity (energy and angular regolution) of the experimental apparatus and the quantities observed-- For example, the extent: to. which radiation of two outgoing photons is excluded. (It is lateresting to note that Compton scatering itself. is a.radiative correction to photoeffect, which must be calculated included ' $1 \pi$ a calculation of quantum clectrodynamic corrections to atonic photoeffect, particularily in the case of one soft outgoing photon.[3]) Photoeffect, scattering and pair production are the three-processes-primarily responsible for the attenuation of electromagnetic radiation in matter. Typically, for a given element the photoeffect dominates at low photon energies, scattering at internediate energies, and pair production at high energles. In Fig. 1 we-see-that for the highest atomic nusbers $(2>92)$ scattering dominates the attenuation of radiation In matter only in the range from about $.8 \mathrm{lleV}$ to 4 lieV. [2] The range broadenes as 2 decreases, beconing 0.1 HeV to 10 $\mathrm{MeV}$ for 1ron, whlle finally, for hydrogen, incoherent scattering dominates the total cross section from a few keV to 100 :leV. $\{2,4\}$

In Compton scattering from bound electrons the electron which scattered the photon is ejected from the atom; in the case that the efection was from an inner shell of a high 2 elenent shortly 
after the ejection (10[-16] sec.) an outer electron makes a transition into the unfilled shell. thereby emitting characteristic radiation. (Filling of vacancies may also proceed through Auger transitions. For very large $Z$ and for electronic transitions between the innerwost shelis, the Auger effect. is less likely than the emission of $x$ rays. [5]) In princlple. one can observe the following aspects of Compton scattering: the energy (wavelength). nomentum- (scattering angle), and polarization of the scattered photon; the energy, momentum (scattering angle), and spin polartzation of the ejected electron; and the energy. nomentun, and polarization of the characteristic $x$ ray. Although, in principle, all of the above ouantitfes are neasurable: in practice, only a few of the observables are measured. In this report we shall not consider observations of any of the polarization properties of the incident or final photons or ejected electrons. The most detailed type of experiment usually performed ceasures the energy and direction of the scattered photon in coincidence with the cheracteristic $X$ ray evicted during the filling of the vacancy, thereby recording from which shell the electron was ejected. Such measurements usually involve inner shells of high $Z(z>47)$ elements at high photon energies. Another common class of experiments.measures the energy and direction of the scattered photon without observing the ejected electron. This class of experiments has gencrally been performed on low $2(2<30)$ elements for incident photon energies several times the $K$ shell binding energy of. the scatterer. A third class of experiments measures only the direction of the scattered photon. In this case both Compton scattering and Raman scattering contribute to the mes iured process. The least detailed experimental arrangement only measures the atcenuacion of radiation in natter, including photoeffect, scattering, and pair production processes.

The nost widely used theoretical treatments of Conpton scattering are based on the impulse approximation and the 
Incoherent scattering factor approxination. The injulse approxination relates the momentun distribution of electrons in the scatterer to the energy spectrum and direction of the scatcered radlation, by contrast, the incoherint scattering factor approxination is generally used to describe tie class of experinents measuring only the direction of the scattered radiation.

Recently, resonances have been ciserved for the Compton scattering of low energy photons, photons whose inttial energy is slightly greater than the $K$ shell binding energy of the scatterer. These resonances, not predicted by the above mentioned theories, ocsur when the incident photon $w_{1}$ efects an electron from the shell while an outer shell electron fills the $x$ hole enitting a phot on of energy $\omega_{2}=E_{K}-E_{2}$ (where $E_{K}, E_{2}$ is the $k$ shell, outer shell binding energy) in the process. This effect has been termed Resonant Raman scattering.

We begin in the following section (Sec. II) by discussing Compton scatzaring from free electrons, which one anticlpates does serve as a higit energy linit of scattering from bound electrons once binding energies are snall in comparison with photon energies. The various tseories for Compton scattexing from bound electrons and the approximations needed in their derivation are discussed in Sec. III. In Sec. IV we review the nature of the avallable experimental results and the accuracy with which the theories apree with experiment. . Resonant Raman scattering.. and other low enesgy Cowpton scattering ef fects are discussed in Sec. V. We summarize our understanding of this process in Sec. VI and indicate certain needs for further work. 
In photon scattering from electrons, if the initial photon eners:y is very much greater than other energies involved in the scat:tering process, one anticipates that the process may be described as scattering fron free stationary electrons. This description is easlest to understand, so we present it first.

then a photon is scattered fron a free stationary electron, the electron acquires some energ: and nomentum from the photon. hence the final photon is shifted in energy (wavelength) fron its initial value. Compton $[6]$, by considering the relativistic equations for conservation of energy and momentum and assuming an initial stationary free. iectron, derived the shif: in wavelength of the scattered photon

$$
\Delta \lambda=\frac{h}{n c}(1-\cos \theta) \text {. }
$$

where $\theta$ is the angle at which the photon is scattered. The shift in wavelenoth of the scatfered photon is completely deterained by the scattering angie. This result predicts that at a given scattering angle only one wavelength will be observed.

Early experimenters[7] using $X$ rays on retal foils observed that the scattered radiation was not a sharp line whose position was conpletely determined by the scattering angle as in Eo. (1). but rather that the line nas shifted frot the value given in Eq. (1) and that the line had a broad shape. These features, explained by Du:fond. 181 result from the inftal mowentun distribution of the bound and conduction electrons.

Assuming the electron is free but. roving. one can show that for small initial electron nomenta the shift in wavelength is given by

$$
\Delta \lambda \sim \frac{h}{n c}(1-\cos \theta)-\frac{2 \lambda \sin \theta / 2}{n c} p_{z} \text {. }
$$


where $\lambda$ is the initial photon waveleneth and ${ }_{z} z$ is the copponent of the fnitial momentual of the electron narallel to the initial photon.

The relativistic cross section $\mathrm{d} d / \mathrm{d} \Omega$ for compron scattering frow a free electron was first calculated by Klein and lishina and by Tam, $\{91$ to lowest order in $a$. The differentlal cross section for unpolarized electrons in the rest svstem of the incident electron $15|10|$

$$
\frac{d \sigma}{d \Omega}=\frac{a^{2}}{4 \pi^{2}}\left(\frac{\omega_{2}}{\omega_{1}}\right)\left(\frac{\omega_{2}}{\omega_{1}}+\frac{\omega_{1}}{\omega_{2}}+4\left(\bar{E}_{1} \cdot \bar{\varepsilon}_{2}\right)-2\right),
$$

where

$$
w_{2}=\frac{\omega_{1}}{1+\left(\omega_{1} / m\right)\left(1-\cos ^{\theta}\right)} \text { i. }
$$

where $\omega_{1}$ and $\omega_{2}$ are the energies of the initlal and scattered photons, respectively. In the low energy limit of $\omega_{1} \rightarrow 0$ this reduces to the classical Thomson scattering fornula

$$
\left(\frac{d \sigma}{d \Omega} T=\frac{a^{2}}{\pi_{1}^{2}}\left(\bar{\varepsilon}_{1} \cdot \bar{\varepsilon}_{2}\right) .\right.
$$

As the scattering angle $\theta \rightarrow 0, w_{2} \rightarrow w_{1}$ and we find the Thonson cross section to be valid for all energies in the forward direction.

Several experiments at very high energies (photons with energies over $0.1 \mathrm{GeV}$ ) have tested total and angular distribution cross sections of Compton scattering and found agreenent with the Klein - Kishina result to an accuracy of 10 to $15 \% \cdot[11-15]$ At such high energies radiative corrections to single Compton scattering and double Compton scattering must be accounted for. These effucts have been calculated by Brown and Feynan, [16] Anders, [17: llork, [18] and Ran and Wang. [19]

Expressions for the Compton scattering from a thermal distribution of free electrons have been given by fibberfors[20] and llamada and kakamura. [21] These expressions are an evaluation of the effective "Wedn-Kishina cross sections of electrons in thermal. 
equilibriun at a givea electron temperature. The plasma described is aptically thin, in that the scattering from individual electrons is incoherent. 
III. Compton scattering from bound electrons

A. Lionrelativistic Formalism

Since most theoretical work to date is based on nonrelativistic quantum mechanics. and the. general feacures are, in fact, included in such a nonxelativistic description, we first distuss the nonrelatfvistlc treatment of Compton scattering from bound electrons.

Nonrelativistically, in semi-classical radiation theory (considering the interaction of the atom.wth the radiation as a perturbation), one replaces the momentur $\bar{p}$ in the Haniliconian by $\overline{\mathrm{p}}-\mathrm{e} \overline{\mathrm{A}}$. The interaction Hamiltonian then contains two distinct teras [22]

$$
\|=e^{2-2} A / 2 m-e-\bar{A} / m r
$$

In Eirst oxder pertubation theory the $\bar{p} \cdot \bar{A}$ tern describes the enission and absorption of phorons. The $\bar{A}^{2}$ tern in first order partubation theory and the $\bar{p}-\bar{A}$ term in second order pertubation theory describe the scattering of a photon.

The differential cross section for Compton scattering by a bound electron may re written[23]

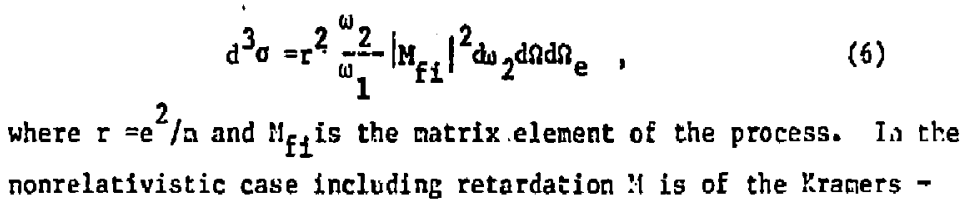
nonrelativistic case including retardation
lleisenberg - Waller type, $[24,25]$ given by

$$
\begin{aligned}
& y=\left.\left(\bar{\varepsilon}_{1}+\bar{\varepsilon}_{2}\right) f\right|^{1\left(\bar{k}_{1}-\bar{k}_{2}\right) \cdot \bar{r}} \mid 1 s
\end{aligned}
$$

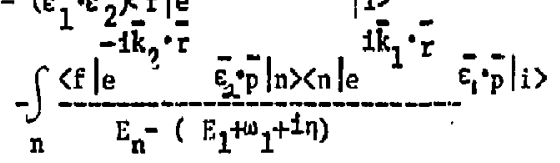

$$
\begin{aligned}
& -\int_{n} \frac{\left\langle\left. f\right|^{1 \bar{k}_{1} \cdot \bar{r}} \bar{\varepsilon}_{i} \bar{p} \mid n\right\rangle\left\langle\left. n\right|_{e} ^{-1 \bar{k}_{2} \cdot \bar{r}}\right.}{E_{n}=\bar{E}_{2} \cdot \bar{p}|i\rangle}
\end{aligned}
$$


where $\bar{k}_{1}$ and $\bar{k}_{2}$ are the ciomenta of the intial and final photons, and $\bar{\varepsilon}_{1}, \bar{\varepsilon}_{2}$ their respective polarizations; $\bar{p}$ is the asympotic momentum of the ejected electron; $\bar{p}$ and $\bar{k}_{2}$ refer to the solid angles in $\mathrm{e}$ and $d \Omega$, respectively. Subscript 1 denotes the initial atcilc state (energy. $E_{1}$ ) and $f$ tenotes the final state of the acom and ejected electron (energy $\varepsilon_{2}$ ). The infinitesirial positive quantity $n$ appearing in the denominacor of the first sum prevents the occurance of an unphysical singularity when the Initial photon excites the electron into an tntermediace continuum state with energy $E_{2}=E_{1}+w_{1}$. Energy conservation tequires that

$$
E_{1}+\omega_{1}=E_{2}+\omega_{2} \text {. }
$$

Momentum is not conserved in the process as described here, since momentum will also be transfered to the nucleus of the atom. Therefore the directions of the outgoing photon and electron are not uniquely determined by momentum conservation arguements. Their energies can vary continuously from zero to upper linits fixed by Eq. (8), is contrast to the sharp angular dependent values they have when the initial electron is taken to be free.

The expression for $\mathrm{Mf}_{\mathrm{f}}$ in Eq. (7) is exact, to lowest order in the fine structure constant $a$, within nonrelativistic quantum wechanics, for a hydrogenlike atome, In the case of a many electron atom, described within a. central.self-conslstent field approxination (the atomic electrons: and the ejected electron move in the same central field in the initial, intermediate, and final states), when the scattering involves a one-electron transition, the matrix element is again.given by Eq. (7). [23]

\section{B. Ilydrogenlike Case}


internediate states in teros of mowentun space intograls involving the cireen's function for the Coulonb fleld, Cavrila\{23] obtained an analytic expression, in terms of generalized hypergeonetric functions, for the matrix element given in. Eq. (7) for $k$ shell electrons. Gavrila and co-workers in a series of papers extended this work by giving expressions for the patrix element in the dipole appreximation (for incldent and final photon energles sufficiently small so that the exponentials in Eq. (7) can be replaced by 1) for scattering by both the $x[26,27]$ and $I[28,29]$ shells. In the dipole approximation, if the outgoing electron is not observed, and if the photon polarizations are not observed, the relavent cross section is

$$
\left(\frac{d^{2} o}{d \Omega d \omega_{2}}\right)=\left(C_{1 j}+c_{2 j} \cos ^{2} \theta \quad / 2 E_{j},\right.
$$

where $j$ refers to the subshell from which the electron was ejected. This yields the shape of the scattered spectrum for every scattering, angle. A further integration will give the over-all spectra: distribution of the photons scattered into all space

$$
\left(\frac{d \sigma}{d u_{2}}\right)=2 \pi\left(C_{1 f}+C_{2 j} / 3\right) / E_{j} .
$$

The values of $c_{1 f}$ and $c_{2 f}$ have been tabulated in Ref. 26 and 27 for the $K$ shell and in Ref. 29 for the $L$ shell.

For the $\mathrm{K}$ shell Gavrila predicted that for srall incident photun energies, where the dipole approxination is valid, the angular distribution is isotropic. In this regine the watrix element together with the cross section of Eq. (9) decrease as $1 / \omega_{2}$ as $\omega_{2}$ increases fromzero. The decrease is monotonic up to the cnt of the spectrum.

For larger incident photon energtes, the first tern of Eq. (7) becomes more and more important. For not too small scattering angles, this term produces a maximum in the cross section $d^{2} \sigma / d \Omega w_{2}$, near the Compton frequency for scattering by free electrons. This maximum moves toward lower frequencies as the 
angle increases. This broad maxinu, related to the romentut listribution of the scattering electrons, contrasts with the sharp behavior of the klein - lishina cross section where $\omega_{2}$ is uniquely deternined by the incident photon energy and scattering angle. The low energy end of the spectrum $\left(\omega_{2}\right)$ 0$)$ is not doninated hy this broad maximum, but rather is dominated by a $1 / \omega_{2}$ behavior. This 1 s an aswect of the linfrared divergence of quantum eloctrodynamics. $130 j$ Between these two features there exists a plateau which is a result of the constructive interference between their natrix elenents.

The $L$ shell scattering also exhibits the low enersy $\left({ }^{\omega_{2}}>0\right)$ infrared divergence of the spectrum and the broad naximum near the Conpton frequency for scattering by free electrons. But even in the dipole apdroximation, the spectrum for scattering fron $2 p$ electrons also exhibits a resonance. This resonance accurs when the incident photon energv is larger than the $K$ shell binding enercy and the scattered photon has an energy of $\omega_{2}=E_{K}-E_{L}$ corresponding to a bound-bound transition. At this energy the denominator of the second sum in Eq. (7) vanishes (In this aproximatica we have neglected the level widths of the electron states). If the numerator does not also vanish (the numerator does vanish for dipole scattering from the $2 s$ subshell, essentially because the is - 2s transition is forbidden in dipole approximation). a resonance occuls. This resonance feature is not restricted to the $2 p$ case, it will be present for scattering fion other subshe?1s.

The total intensity of photons scattered into a certain angle slould be calculated by integrating Eq. (6) over both the outgoing electron angle and the outgoing photon energy. Th1s should yield the analog of the Klein - Mishina cross section for bound electrons. Ilowever, because of the $1 / \omega_{2}$ behavior of the matrix clement as $\omega_{2} \rightarrow 0$, do/d $\Omega$ does not exist. To accurately calculate this quanitity, one must include the radiative corrections to the Compton process. One such correctlon is double conpton scattering, 
where a second soft photon is also enitted during the scattering event. This demonstrates that when one idants to calculate $d \sigma / d \Omega$. the attenuation of a flux of photons passing through matter withont observing the scattered phuson energies and angles, the contribution of Compton scatteriog from bound electrons cannot be considered alone. To obtain such a cross section, one must also add the contribution of photoeffect, including the first-order radiative corrections to atomic photoeffect. [3]

\section{The $\Lambda^{2}$ Approxination}

Except for the woali of Cavrila, all nonrelativistic treatments of Compton scattering by bound electrons which have been given so far are based on the $\Lambda^{-2}$ approximation of the matrix elenent : $f i$, that is

$$
\left.u_{f 1^{n}\left(\varepsilon_{1} \varepsilon_{2}\right.}\right)\left\langle f\left|e^{i e^{-} \cdot \bar{r}}\right| i\right\rangle .
$$

Eisenberger and Platzman[31] have argued that if $\omega_{1}$ and $\omega_{2}$ are auch greater than $E_{n}-E_{1}$, then Eq. (7) may be approxinated by

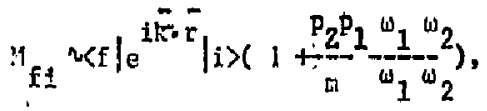

where all polarization factors have been dropped. The $\bar{p} \cdot \bar{s}$ contribution (the second part of Eq. (11)) is small in conparison ro the $A^{-2}$ contribution in this limit. In fact, for stationary initial electrons $P_{1}=0$ and the contribution vanishes. However, at low energies the situation is reversed, since the $\vec{A}^{2}$ tern of Eq. (7) vanishes in the dipole approximation, whereas the $\bar{p} \cdot \bar{A}$ term Joes not. In the limit $z \rightarrow 0\left(E_{1}>0\right) E q .(10)$ becomes exact and yields the nonrelativistic version of the klein - Kishina cross section. 
Lsing hydrozenlike wave functions for both the initfal and final wave functions and assuming unpolarized radation, Schaldt [32] and Sujowski and liagel[33] abtained the $\bar{A}^{-2}$ approxibation for the incoherent scattering of a photon into the enersy interval $d_{42}$ and solid angle $d \Omega$ by a $k$ shell electron, where the final electron is represented by a continuum hydrogenlike state with a definite asymotic electron monentun, as

$$
\begin{aligned}
& \frac{d^{2} \sigma}{d n d \omega_{2}}=\frac{r^{2}}{2}\left(1+\cos ^{2} \theta\right) \frac{256 a^{6 \omega_{1}}}{3}-\frac{2}{w_{1}} \frac{q^{2}\left(a^{2}+p^{2}+3 q^{2}\right)}{\left(a^{2}+(n+a)^{2}\right)^{3}\left(a^{2}+(p-q)^{2}\right)^{3}} \\
& \exp \left(-\frac{2 a}{p} \tan \frac{-2}{a}-\frac{2 p a}{a^{2}+p^{2}+q^{2}}\right. \\
& x-1-\exp (-2 \pi a / p) \text {. }
\end{aligned}
$$

here $q=\left(\omega_{2}^{2}+\omega_{1}^{2}-2 \omega_{1} \omega_{2} \cos \theta\right)^{1 / 2}$ is the monentum transfered to the atom, $\theta$ is the angle between $\bar{k}_{1}$ and $\bar{k}_{2}, \bar{p}$ is the momentur of the scattered electron, by energy conservation: $p^{2} / 2=w_{1}-w_{2} a^{2} / 2$, and $a=a z$.

For not too small scattering angles this result yields a scattered szectrum which exhibits a broad naxinum centered about a line shifted from the free conpton frequency toward the energy of the Incident photon. At saller angles energy conservation cuts off the spectrum before it aciieves Its maxinum. Since tiis is an $\mathrm{A}^{2}$ approxiwation the spectruin goes to zero as $\omega_{2}$ goes to zero. By numerically integrating Eq. (12) over the sagnitude of the electron momentun. they obtained a nunerical result for do/dl . which is a function only of $q^{\prime}=a / a$. This cross section is zero at $q^{\prime}=0$ and increases Lonotonically as $q^{\prime}$ increases aporoaching 1 for large $q$ " $\left(q^{\top}>3\right)$.

Shinizu et. al.134] have derived an expression for the incoherent scattering of a photon into the solid anele $d \Omega$ by a $\mathrm{K}$ shell electron. Thev neglected transitions into cxcited discrete states. used a Coulomb wave function for the initial $\mathrm{K}$ shell electron and a plane wave for the ciected electron, and integrated 
over all possible final states, and obtalned

$$
\begin{aligned}
& \frac{d \sigma_{u}}{d \Omega}=s \frac{d \sigma_{E}}{d \Omega} .
\end{aligned}
$$

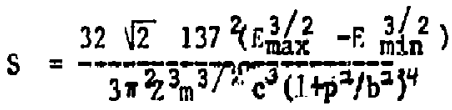

where $D$ is the initial monentum of the $F$, shell electron $\left(p=\left(2 m E_{K}\right)^{1 / 2}\right)$, and $b$ is $2 h / a_{0}$, where $a_{0}$ is the first Bohr radius. $E_{\max }$ and $E_{\min }$ can be estimated as

$$
\begin{aligned}
& E_{\text {max }}=T+\left|E_{K}\right|+2\left(T \mid E_{k}\right)^{1 / 2} \\
& E_{\text {min }}=T+\left|E_{K}\right|-2\left(T \mid E_{k}\right)^{1 / 2}
\end{aligned}
$$

where $T=k A(1-\cos \theta) /(1+\lambda(1-\cos \theta))$ and $\Lambda=k / \mathrm{mc}^{2}$. anr oo $j i d \Omega$ is the klein - Nishina cross sectlan. This result pr.dicts a zero differential eross section at $\theta=0$, and the crass section rises monotomically as $\theta$ increases.

The fac: that the tatrix element goes to zern as the scattering arole decreases is a general feature of all $\vec{A}^{2}$ based approxinatisss. The feature is a consequence of the orthozonality of the wave tunctions. At stall scattering angles, the momentum transfer to the tareet electron tends to be small connared with their initial momentum. ant there is a small probabllity that the aton w11l absorb enough energy to renove the target electron.

\section{Incohement Scattering Factor}

A11 present theoretical tabulations[35-39] of the differentiat cross section $d$ o/d $\Omega$ of photons scattered from atoms are based upon the incoherent scatterins frinction. Since the energy of the scattered photon is not observed, the dffforential cross section must Include both excitation and Ionization of the atom, and must be summed over all posgible final states. In an independent 
particle picture, the excitation or lonization involves one electron only, leaving the other electrons undisturbed. Thus the Incoherent scattering function for an atom represents an average of the incoherent scatcering functions for its separate e ectrons. The incoherent scattering approximation starts from Eq. (10) and makes the following further approxiarations:

(1) In the differential cross section

$$
\begin{gathered}
\left.\frac{d^{2} \sigma}{d \Omega \omega_{2}}=\left(\varepsilon_{1} \varepsilon_{2}\right)^{2} \frac{\omega_{2}}{\omega_{1}} \sum_{\mathrm{f}}\left|<E \sum_{j} e^{i\left(\dot{\mathrm{k}}_{1}-\overline{\mathrm{k}}_{2}\right) \cdot \bar{\tau}_{j}}{ }_{i}\right|\right\rangle^{2} \\
\quad \times \delta\left(E_{2}+\omega_{2}-E_{1}-\omega_{1}\right),
\end{gathered}
$$

one replaces $\left(\bar{k}_{1}-\bar{k}_{2}\right)$ by $\left(\bar{k}_{1}-\omega_{2}{ }^{0} \hat{k}_{2}\right.$ with $\hat{k}=\bar{k} /|k|$, and $\omega_{2}$ by $\omega_{2}^{0}$, where $\omega_{2}{ }^{0}$ is the nean of $\omega_{2}$, then one san analytically integrate over ${ }_{2}$, and usually one takes $\omega_{1}$ or $\omega_{2}{ }^{0}$ as $\omega_{2}{ }^{0}$, where $\omega_{2}{ }^{0}$ corresponds to the (free) Compton frequency iq. (4). This approxination is equivalent to asuning that the main contribution to the integral over $\omega_{2}$ is from the region immediately surrounding the free Coapton frequency.

(2) One assumes that the initial state and all possible final states fora a complete set, and invokes the closure property. This ignores the fact that transitions from the initial state to occupied bound states are forbidden and not all continum states are accessible due to the requirements of energy conservation. Then one obcains, on integration ovér scattered final photon energies w 2 '

$$
\frac{d_{\sigma}}{d^{\Omega}}=\left(\frac{d_{\sigma}}{d_{\Omega}}\right) \frac{w_{2}^{2}}{w_{1}}\left(\sum_{i, j}\left\langle i\left|e^{1 \bar{q} \cdot\left(\overline{r_{1}}-\overline{r_{j}}\right)}\right|_{i}\right\rangle-F\left(q^{2}\right)^{2}\right),
$$

where $F\left(q^{2}\right)$ is the atomic form factor,

$$
F\left(q^{2}\right)=\sum_{n}\left\langle i\left|e^{1 \bar{q} \cdot \bar{x}}\right| i\right\rangle,
$$

and $\bar{q}=\bar{k}_{1}-\bar{k}_{2}$. (Note that in the $\bar{A}^{2}$ approximation, unlike the core exact infrared divergent full calculation, do $/ \mathrm{d} \Omega$ does exist.) 
The incolerent scattering function is equal to zero at $\theta=$ ?, due to the orthogonality of the waye functions. As the scattering angle increases the incoherent scatcring function increases, becoaing one for large momentum transfers. Eq. (16) has been evaluated ustng various types of wave functions (llerman-Skillnan, liartree-Fock, configuration interaction, etc.). The values obtained by the different evaluations vary by several per cent.

\section{E. Impulse Approxination}

The most commonly used theory to describe the spectral distribution of photons scattered from an atom $d{ }^{2} \sigma / d \Omega d \omega_{2}$ is the iapulse approxination. This theory depends upon the assuption that the energy transfered to the electron is large, so that electron binding energies can be neglected.

The case of a photon scattcring frow a free stationary electron ias discussed in Sec. II. In this process the energy of the scatcered photon is completely determined by the scattering angle and incident photon energy Eq. (4). If the electron is free but not stationary, the resulting cross section is given as [32]

$$
\frac{d \sigma}{d a}=\frac{r^{2}}{4 r^{2}\left(1-\cos ^{a} \gamma^{2}\right.}\left(\frac{\omega_{2}}{\omega_{1}}\right)\left(\frac{\omega_{1}}{\omega_{2}}+\frac{\omega_{2}}{\omega_{1}}-2+4\left(\bar{\varepsilon}_{1} \bar{\varepsilon}_{2}\right)\right),
$$

where

$$
\frac{\omega_{2}}{\omega_{1}}=\frac{1-\beta \cos \alpha}{1+\omega_{1} / a(1-\cos \theta)-\beta \cos \alpha}
$$

The angles and $a$ are the angles between the incident electron and the incident and outgoing photon, respectively, and

$$
|p|=\beta \gamma m, \quad \gamma=\frac{1}{\sqrt{1-\beta^{2}}}
$$

The angle $\phi$ is the angle between the rlanes forced by $\bar{k}_{1}, \bar{p}$ and $\bar{k}_{1}$, 
$E_{2}$ The angle $a^{\prime}$ cant be expressed in terms of $\alpha, \theta$, $\phi$ :

$$
\cos \alpha^{\prime}=\cos \alpha \cos \theta+\sin \alpha \sin ^{\theta} \cos \phi .
$$

If the electron does not have a single well defined monentun, but rather a nomentun distribution as in the case of a bound clectron, the scattered photon energy will not be a single frçuency at a given scattering angle. The momentun distrihution of the scattering electrons. will produce a distribution of frequencies at each scattering angle.

By semiclassical arguenents Du:lond[8] related the spectral distribution of the scattered radiation to the nomentum distribution of the bound electrons. IIs results were rederived and refined within tihe framework of quantun mechanics by Eisenberger and Platzman[31] and by Kilby.[40] The inpulse approxination follows from the $\tilde{h}^{2}$ approxination and further assumes that the scattered electrons are free (but nitoving) during the entire collision proces5.141] Refore the collision let the electron have mowentur ? 1 and after the collision $P_{2}$. Then

$$
\begin{aligned}
& E_{1}=p_{1}{ }^{2} / 2 \\
& E_{2}=p_{2}{ }^{2} / 2 .
\end{aligned}
$$

Conservation of nomentum for this frce electron case gives

$$
\bar{p}_{2}=\overline{p_{1}}+\bar{k} ; \quad p_{2}{ }^{2}=p_{1}{ }^{2}+2 \bar{k} \cdot \bar{p}+k^{2}
$$

and the delsa function in Eq. (15) becones

$$
\delta\left(\left(2 \tilde{k} \cdot \bar{p}+k^{2}-\omega\right) / 2\right)=\delta(k(\bar{k} \cdot \bar{p} / k-(\omega / k-k / 2))) .
$$

lithin tise impulse approximation the final state is taken to be a plane wave

$$
|\mathrm{f}\rangle=\mathrm{e}^{\overline{\mathbf{i p}} \overline{\boldsymbol{r}}} .
$$

The monentum space bound state wave function is the Fourier transform of the corresponding position space function so that 


$$
x_{1}\left(\bar{p}_{1}\right)=(2 \pi)^{-3 / 2} \int e^{-1 \bar{p} \cdot \bar{r}} \psi_{1}(r) d^{3} r
$$

and the sum over final states is

$$
\sum_{\tilde{f}}->\int(2 \pi)^{-3} d^{3} p_{2}
$$

Choosing $\vec{k}$ to 1 ie in the $z$ direction and using momentum conservation

$$
\begin{aligned}
& \sum_{f}\left|\left\langle\mathrm{f}\left|\mathrm{e}^{\mathrm{i \overline { \textrm {k } } \cdot \overline { \mathrm { r } }}}\right| \mathrm{i}\right\rangle\right|^{2} \delta\left(E_{2}-\mathrm{E}_{1}-\omega_{1}+\omega_{2}\right) \\
& =\int d^{3} p_{2}\left|(2 \pi)^{-3 / 2} \int d^{3} r a^{1 \bar{p} \cdot \bar{r}} \psi_{1}(r)\right|^{2} \delta\left(k\left(p_{12}-(\omega / k-k / 2)\right) \text { (20) }\right. \text {. }
\end{aligned}
$$

Defining $q=w / k-k / 2$. Eq. (20) reduces to

$$
\left.\left.\int d^{3} p_{2}\right|_{1} ^{x}\left(p_{1}\right)\right|^{2} \delta\left(k\left(p_{1 z}-q\right)\right) .
$$

Foting that for $\omega_{1}, \theta$ and $\omega_{2}$ fixed, $k$ is fixed as well, it follows that the intepral over $d^{3} p_{2}$ is equivalent to an interration over $d^{3} p_{1}$. :laking use of the properties of the delta function Eq. (2I) reduces to

$$
\int_{-\infty}^{\infty}\left|x_{1}\left(p_{1 x}, p_{1 y}, q\right)\right|^{2} d p_{1 x} d p_{1 y}
$$

which is equivalent to an integration over the plane In monentum space $P_{12}$. For a momentum density which is spherically symetric, 16 is convient to rewrite $E q$. (21) in cylindrical coordinates where

$$
J(\eta)=2 \pi \int_{(q)}^{\infty}\left|\psi_{1}\left(p_{1}\right)\right|^{2} p_{1} d p_{1}
$$

This becomes the result for the Compton profile in the Inpulse apptoxination

$$
\frac{d^{2} \sigma}{d \Omega d \omega_{2}}=\frac{d \sigma}{d \Omega} \frac{T}{k} \frac{r^{2}}{\omega_{2}} \frac{\omega_{1}}{\sigma^{2}} J(q) .
$$

The impulse approximation is equivalent to a photon scattering inelastically from a free electron gas with a spherically syonetric momentum distribution where both energy and nomentum are conserved 
during the collision. The nomentum density for any particular ronentum in the free electron gas is obtalned from the square of the fourler transform of the initlal bound state radial wave function. The energy-monentum conservation zelat lons determine that for an Incldent photon with energy $w_{1}$ scattering at any fixed $\bar{k}$, contributions to the scattering from electrons with mamentuth $\bar{p}$ can occur only if the projection of $\bar{p}$ on the scattering vector $\bar{k}$ equals $q$. This can occur if $\mid \overline{p l}$ is greater than or equal to $q$. The Coapton profile is the projection of the electron nonentun distribution nnto the scattering vector, the one-dinensional momentum distribution function. The integration of Fq. (23) over $w_{2}$ will yleld a quantcy which is comparable with the ircoherent scattering factor. We have not found any discussion of this point.

In a simple description of photons scattering from a metal foil, the Compton profile $J(q)$ is the sun of two distinct components. A broad background die to the core electrons. And. if the conduction electrons are treated as free in which all states below the Fersi vomentum are occuafed and all those above it are cmptr. the cotpton profile is the orniection of this distribution (a uniforn ssinere) orto a dingeter. a oarabola. Thus the orofile for the foil is the sua of the two contrtbutions. The area under each component curve is pronortional to the number of electrons of that trpe. Realisticallv. the conduction electrons are not strictlv free and hence their contrlibution to the profile will not be a sicole inverted parabola.

lluch of the work on Compton profiles has concentrated on usine more accurate bound state wave functions in the calculation. For a good discussion of Compton profiles see the book Compton Scattering bv 3. Willans/42] and the review articles by Cooper. [22.431 In Darticular. Drofiles have been calculated using Hartree-Fock wave functions 144$\}$, relativistic llartree-Fock wave functions $[45\}$. correlated wave functions. 146.471 and renoroalized free aton trodels. 1481 Such calculations vield results differing from each other by as much as 20\%. but the basic valloitr of using such wave 
functions in a morelativi tic sinele narticle fornalisn is an osen oustion.

Although the joulse anoroximation fs usually used to cescribe ohoton scattering from an atom. exoressions have been eiven for scattering fron certain individual subshells, in particular for scattering from the : shell of a hydrogenlike atom

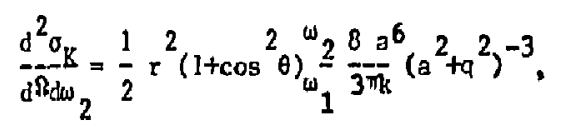

Various investigators $[31,49]$ have examined the effects of repcesenting the $c$ jected electron by a plane wave instead of a hydrogenlike continuum wave. These investigators have begun with Eq. (15), represented the final electron by a continuun Coulomb wave where $\mathrm{E}_{2}=\mathrm{p}_{2}{ }^{2} / 2$. The sum over final states is renlaced by an Integral over the romentum of the oukgoing electron. This quanity is then averaged over the directions of the electron monentun. Dividing by the Thonson cross section and the ratio of photon energles, they obcain an expression for the "exact" Coapton profile. For weak binding and high incident photon energics the two resulis are sinilar $\left(\omega_{1}-100 E_{B}\right)$. As the scattering eiseray decreases the agreenent worsenes. In many cases, using Coulonb wave functions rather than the plane wave for the continuum, secondary zidxima occur which have been related to nodes in the bound state monentum wave function. Ilowever, for Couloab wave functions such secondary maxima axe too smalt to be observed in the sun of cross section profiles over all subshelis in an aton.

\section{F. Relativistic Approaches}

For the most part, attempts at relativistic calculations of the Cofipton cross sections have involved evaluating the $\mathrm{A}^{-2}$ natrix element of Eq. (10), [5n-53] the incoherent scattering function, and 
the inpulse approxination using relativictic wave functior . It is not clear whether such an evaluation is justificd. (Such calculations rely on the fact that the nonrelativiste $\bar{p} \cdot \bar{n}$ tera af the vatrix element is small at high energy, but this need no longer be the case in the reiativistic dowain where $\bar{p}$ is not snall.) Schunacher[52] has extended the work of Randles [50] obtaln, ng ar. expression for $d^{2} \sigma / d \sqrt{d} d{ }_{2}$ for all s-subshells of the atom. This is an $\bar{A}^{2}$ approximation evaluated using relativistic Coulomb wava functions for the initial and final electron. Ne displayed resulis for incident photons of energy $662 \mathrm{keV}$ scattering from lead into internediate angles. To account for the shielding of the nuclear charge, effective charge numbers were used for the $L_{I}$. ${ }^{\prime} I_{I}$, and $l_{I}$ subshells; no shielding corrections were used for the : sheil. liis results for the energy spectra begin at very snall values at $w_{2}=0$, have a naxigum at energies slightly greater thon the enersy for scattering by free clectrons, and dtop abruptly to zero at $\omega_{2}=\omega_{1}-\varepsilon_{B}$, where $E_{B}$ is the binding anergy of the electron. The shift of the caxinum relative to the energy for frce electrons decreases at increasin principal number. The curves obtained for the $L_{I}$. " $I$, and $l_{I}$ subshells have local minima close to the Compton max: These ninima are related to the structure of the s-wave furctions.

Pradoux et. al. [53/have evaluated the $A^{-2}$ approximation usins, a Coulomb $\mathrm{k}$ shell wave function for the initial electron and a Coulonb partial-wave series for the final electron for a variety of 2 's at internediate scattering angles and for $\omega_{1}=60$ and $662 \mathrm{keV}$. For the low 2. $(2=32)$ calculation at high energv, the maximum of the spectrun is shifted frow the free Compton cnergy toward lover energies, for high $Z(Z=82)$ at high energy the maximun is shifted toward higher energies.

A more consistent relativistic treatment would involve the evaluation of the serond order S matrix. in the Furrv alcture[54]. Compton scattering is described by the second order $S$ matrix elerient 
where

$$
s_{f t}=s_{t 1}^{(a)}+s_{f 1}^{(e)}
$$

$$
\left.\frac{s_{f 1}^{(a)}=}{8 \pi 2}\left(\omega_{1} \omega_{2}\right)^{-1 / 2} \iint d^{4} x^{\prime} d^{4} x_{2} \psi_{f}\left(x^{\prime}\right) r \varepsilon_{2}{ }^{*} e^{1 k_{2} x^{r}} s\left(x^{\prime}, x\right) r \varepsilon_{1} e^{-1 k_{1} x}{ }_{1}(x)\right\} .
$$

and

$$
s_{f 1}^{(e)}=s_{f 1}^{(a)}\left\{k_{2}\left\langle-k_{1}, \varepsilon_{2}^{*} \longleftrightarrow->\varepsilon_{1}\right\} .\right.
$$

are the anplitudes which correspond to the absorption of the incicient ohoton before enission of the final photon and to the eaission of the final ploton prior to the absorption of the incident photon, respectively. $[55] \mathrm{s}\left(\mathrm{x}^{\prime}, \mathrm{x}\right)$ is the clectron propagator in the external field. Brown et. a1. $\lceil .561$ developed a nethol of solution based upon defining. for a time independent external ficld.

$$
F^{(a)}=\int d^{4} x S\left(x^{\prime}, x\right) \gamma^{-\varepsilon} e_{1}^{-1 k_{1} x} \psi_{1}(x) .
$$

The anplituce $F^{(a)}$ satisfles the inhomogeneous Dirac equation

$$
\left.(i)-k_{1}-E_{1}\right) i^{(a)}=-\tau a_{1} e^{-i k_{1} x} \psi_{1}(x) \text {, }
$$

and

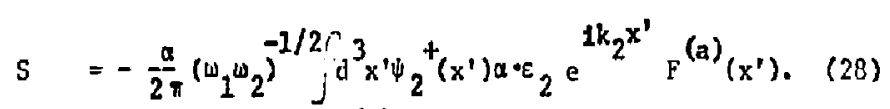

Bv expandine the amplitude $F\left(\mathrm{f}^{\prime}\right)$ and the final electron state in their respective, partial wave series and the final photon in its multipole series, the matrix element mav be written as an infinite sur of radial integrals over the product of radial wave functions and spherical Bessel functions. Brown et. al, applied this nethod to the calculation of Rayleith scattering, and it has since been further developed for Ravleigh scattering s.v. Johnson and Feiock[57] and Kissel and Pratt. [58]

llenry[59] has atten.tted to apply these methods to Conpton scattering. He analytically performed the suruation over hvdrogenic internediate states and perforried the final radial interations nuterically to abtain the differential cross section 
$\mathrm{d} \sigma_{\mathrm{K}} / \mathrm{d} \Omega$. He compared his results to calculations of the incoherent scattering factor for the $K$ shell of lead by scaling his hydrogenic results by $q^{\prime}=q / a z$; $q$ is the momentum transfer, sce Ref. 33).

Thittinghan[60] perfermed a Coulombic nuaerical evalu, tion of $S$ following the approach of Brown et. al. lle completed a set cf calculations for the doubly dfferential cross section $\mathrm{d}^{2} \sigma_{K} / \mathrm{d} n \mathrm{d \omega}_{2}$ of the $X$ shell of lead at $662 \mathrm{koV}$. The energy spectrun, which he calculated, was shifted by $10 \%$ from the free Compton energy toward higher photon energies for scattering at $60^{\circ}$, This shift was ar, lc dependent. It was toward lower energies at larger angles. The lowest scattered photon energy which he considered was $100 \mathrm{keV}$, which was within the region of least accuracy in his calculation. In this region his res:y $s$ did not exhibit a rise in the energy spectra as $\omega_{2}$ decreased. To obtain the differential cross section do $\mathrm{K}^{/ d \Omega}$, he extrapolated his results to the $\omega_{2}=0$ region and numerically iniegrated over $\omega_{2}$ * His calculated cross section ratio $d \sigma_{K} / d \sigma_{F}$ is sontricantly larger than incoherent scattering function calculations.

Wittwer[si] perforsed numerical calculations of $d^{2} \sigma_{K} / d \Omega w_{2}$ for goid at 145 and $320 \mathrm{keV}$ and tin at $145 \mathrm{keV}$. He did not follow the approach of Brown in that he numerically performed the sums over intermediate states and neglected binding effects in the internediate states. He also truncated his calculation at $1=2$ for both the initial and final photon multipole serles, where 1 is the angular toventun of the nultipole operator. His results, clained to be accurate within $12 \%$, displayed a striking infrared divergence. The effect dominates his calculation of gold at 145 keV.

Owen[62] has performed an analytic calculation of the differential cross section and Conpton profile by ignoring the effects of the external field after the initial photon-electron intcraction. In this approximation, the free electron propagntor is used instead of the Coulonb propagator in Eq. (26) and the Final 
electron is represented by plane waves. He then solved the second order $S$ matrix and obtained expressions for both the coubly and singly differential cross sections, $d^{2} \sigma / d \Omega d \omega_{2}$ and $d \sigma / d s_{i}$, in terns of the upper and lower radlal components of the initial electron wave function. These results reduce to the viein-iishina cross section for scattering from free stationary electrons.

C. Comparison of theorecical approaches

Both Tseng et. al. [25] and Pradoux et. al. [53] have made comparisons between the various theoretical results for $x$ shell electrons in a hydrogenlike aton. Tseng, et. al. compared the nonrelativistic $\bar{A}^{-2}$ formula of Schnaidt, [32] Eq. (12), and the nonrelativistic dipole $\bar{p} \cdot \bar{n}$ formula of Gavrila[26] and the hydrogenic inpulse approxisation, Eq. (24), with the minarical results of Whittingham. [60] in Figs. 2-4 we display their comparison for the doubly differential cross section $\mathrm{d}^{2} \sigma_{\mathrm{K}} / \mathrm{d} \Omega \mathrm{d} \omega \mathrm{N}$. lie see that in general the $\bar{\Lambda}^{2}$ approxination and the inpulse approximation are relatively close to each other at all displayed scattering angles and energics. But that the $\bar{p} \bar{A}$ rosults of Gavrila and the relativistic results of thittinghan differ strongly with each other.and with the two $\bar{A}^{2}$ based approximations. In Fig. 5 we display the singly differential cross section $d \sigma / d \Omega$. (where to obtain a meaningful result, the integral over the $\bar{p} \cdot \bar{A}$ term in Cavrila's work was evaluated using a low energy cutoff). At small scattering angles we see that calculations utilizing relativistic wave functions are nonzero at $\theta=0$. At larger scattering angles the discripences cannot be so easily classified. All calculations, except the nonrelativistic $A^{-2}$ and impulse, disagree with each other. They also concluded that in the calculation of total cross sections, the $\bar{\Lambda}^{-2}$ approxination alves very bad predictions for photon scattering near the $k$ edge and 
gives results accurate to luss than $10 \%$ for photon energies larger then 20 titres the $k$ shell binding energy.

Pradoux's work also shows significant differences hetween relativistic and noncelativistic calculations. They compared $k$ shell results of theirs with those of Schnaide, Gavrlla, Whit ingham, and Schutuacher. They reported that the position of the naxinum of the spectra agree in relativisic calculations of Whittinghan, Pradoux, and Schumacher. This position does not agree with the nonrelativistic calculations of Cavrila and Schnaidt. In a11 their cases the full width at half maximum for all nonrelativistic calculations are larger than those for relativistic calculations. Whiteinghain's results are consistently larger than all the other calculations.

It appears that at this point that the varlous theoretical approaches differ siantficantly and that one cannot have any confidence in any of these approaches. 
IV Experinents and comparison to tincrettal wor:

The recent Interest in Compton mensurements, going hack to the 1960 's, has resulted fron technical improvements in computers and detectors. The experinental efforts have been concentrated nainly in two directions. One is to study the detalled proflle $J(q)$ of the Compton line and its relation to the electronic momentum distribution of the scatterer. In this type of experiment a monochromatic, collimated bean of photons is scattered fron the sanple and a energy (wavelength) analysis of the bean scattered through a flxed angle is carried out. This class of experinents has usually been performed on lower 7 eleinents, where it has been believed that the nonrelativistic $\bar{A}^{-2}$ approximation which underlies the analysis is justified.

The other matn experimental direction tries to isolate the contribution to the compton cross section do /dn from individual atonic shells by using favorible experinental setups. The principle of such experinents is always related to the enission of an $X$ ray following the ejection of a bound electron. The $x$ ray, characteristic of the atomic energy level, is emitted shortly after the interaction. When this $X$ ray is abserved in concidence $w i t h$ the scattered photon, the Incoherent scattering event can be associated with scattering frow electrons in a particular shell. This is grnerally the $K$ shell of medium to high $Z$ elements. due to their higher characteristfo eneroies. these characteristic $X$ ravs are easier to detect.

There are also a lintred number of experiments neasuring the doublv differential cross section from the $x$ shell separatelv. or focissing on the total aton cross section rather thait $J(a)$. as well as. measurenents of the singlv differential cross section do $/ d \Omega$ from total atoms. We shall brinfiv comment unon each of these areas of experimentation in this section. hepinnine with the daubly differential cross section $d^{2} \sigma / d \Omega \omega_{2}$ in A and B. and the sirnelv 
difterential cross section in $C$. In each section we examine both the total atom and shell studies.

A. Measurement of $\mathrm{J}(\mathrm{q})$

There have been numerous measurements of the Compton profile $J(q)[42]$, usually on the lower $Z$ elewents $(2<36)$ at incident photon energles between two and 3000 times the $K$ shell binding energy. The most commonly used incldent energtes are 17.4, 59.54, and 159 kev. Measurements of $J(q)$ require substantfal unfoldting of the raw expertmental data. The results $I(q)$ are cyptcally displayed graphically, thereby easily showing the general features: a sharp peak due to the valence or conduction electrons superimposed upon a broad peak due to the inner shell (core) electrons. These measurements on gases, liquids, solids, crystals, and molecules easfly shov tie environmental effects upon the outer or conduction electron acmentum densities. The main thrust of this work has been to determire the wave functions which yield $J(q)$ agreeing with experiments sest.

B. Measurement of $d^{2} \sigma / d \Omega d \omega_{2}$

Using colncidence measurement techniques the energy spectra of Cospton scattering from the $K$ shell of atoms has been reported by Spitale[63], Pradoux et. a].[53], and Kane.[64] Spitale performed measurements on gold $(z=79)$, holmium $(z=67), t \pm n(z=50)$, and iron $(z=26)$ at incident photon energies of 145,320 , and $662 \mathrm{keV}$. Spitale's measurements showed a scatcered peak superimposed on a continuum which appeared to diverge at the low end of the spectrum. The scattered peak was narrower than would be expected from the 
bound state electron inotion, the peak width reached a wairun near $z=50$ and then decreased with increasing atonic nuriber. $"$ is measurements disagreed with littwer's predictions on the behavior of the infraced divergence as a function of scattering angle, and also on the behavior of the broadened peak. Pradoux et, al, have measured the energy spectrut from $C u(z=29), G e(z=32)$, and :to $(z=42)$ at incldent evergies of 60 and $662 \mathrm{keV}$. Within the experimental accuracy, their measurements agree with his relativistic evaluation of the $\Lambda^{-2}$ approxination; predictions for the shift of the maximun and the full width at half naxinum.

Schumacher et. al. $[52,65]$ have measured and reported dircetly the differential cross section d $0 / \partial \Omega \mathrm{d}_{2}$ for $662 \mathrm{keV}$ photons Incident on lead and incident photons at $279 \mathrm{keV}$ on copper, tin, and lead. For the scattering of $602 \mathrm{kel}$ photons, they report - the differential cross section begins at very small values of ${ }_{2}+100$ kel, have a thaxinua at energies slightly larger than the energy for scattering by free electrons, and irop to zero abrupty ac

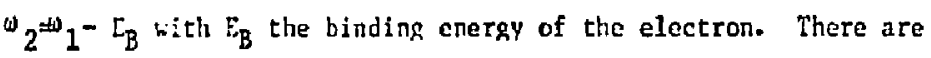
abrupt drens in the differential cross section at 573.6 and $6.46 \mathrm{kel}^{\prime}$ corresponding to the disappearence of the contributions of the ? and $\tau$ she125, respectively. At 279 liev they compared their experinentally neasured differential cross section to an inpulse approxination based upon Coulonb wave functions of an effective charge to represent screening and a nonrelativistic $\mathrm{A}^{2}$ calculation. They observed that at the high energy end of the spectrun there is a discripency of 1.5 in the case of conper and a factor of 4 in the case of lead. which they atcribute to a deviation of the $\mathrm{A}^{-2}$ approxination fron secon? order pertubation theory.

\section{Hleasuresients of do $/ \mathrm{d} n$}

Fost recent wark $\{33,34,66-79]$ on the angular distribution of 
scattered plwtons has involved coincidence reasurenents which Identify fron witich shell the electron was ejected. Due to the cnergv cutoff of the experimental equipment, these experinents do not seasure the divergent part of the cnergy spectrum. Experinents which isolate the the contribution to the compton line by individual atomic shells show the effects of binding upon the scattered cross section. At the large scattering angles, where the energy transfer to the $Y$. electrons is large compared to their initial energv. the cross section for scattering approaches the Conpton cross section for scattering bv free electrons with velocities approaching the initial velocity of the scatterci electron.

Another expected effect of bindine of target elecerons is a lowering of the cross section in the forward direction. in combarison to that for free electrons. $\Lambda t$ small scattering angles. the momentus transfer to the tareet electron tends to be small conpared wit: sient initial rozentum, and there is a small nrobability tiat the aton will absorb enough energy to rentove the tareet elec:son.

In Tabies I - IV. We give cost of the published values of the differential : shell cross section ratio $d \sigma_{R} / d \sigma_{F}$. where $d \sigma_{F}$ is the free electron (Hlein-Mishina) cross section. Table I shows the differential cross section ratios for an incident photon of 279 keV. At this enerr there is no overlao between the exnerinental grouds. each growo has verformed thejr work on a different set of eleibents. It is interestine to onint out that the results ohtalned hv $P$ ineot for Ta $(Z=73)$ are anornximately half of the values olbtained bv :Jurty et. al. for $6(7:=74)$. The experimental results have been crmbared to evaluations of Eos. (12) and (13). as vell as. to averages over ancle of Ea. (19), where the electron is assumed to have a momentum whose magnitude is giver by the binding enerev. All of these theoretical aporoaches differ sisnificartly from the nasured values. Table II shows the cross section ratios for an incident vhoton of enersv 320 kel. The results of the two 
exoerinents are in verv sood aereenent. but ft should te noted that the resulis carie from the sane laboratorv.

The cross section ratio $d \sigma_{\mathrm{K}} / \mathrm{d} \sigma_{\mathrm{F}}$ at $\omega_{1}=662 \mathrm{kel}$ is bv far the nost bildely studied. All this work clearly subports the acneral trends noted by early workers: the lowering of the cross section ratio at stall angles and as the angle tncreases the cross section ratio increases monotonlcally. Put the absolute values for a tiven cross section ratio vary by as much as $40 \%$. The measured values are greater than $k$ shell incoherent scattering factors at small angles and larger than the averaged (over angle) klein-:'ishina for large angles. Pingot's measured values are consistently lower than those of other experimenters, although the general trends are cosparable. These results also strongly disagree with evaluations of [q. (12), (13), and (19). For the case of lead the experirental results have ieen compared to the work of lihittinghan. ihittinghas's predictions lie within the experimental bars of Shaizu et. 21., :lotz and Missoni, and East and Levis; but lie above the ezror bars given by Sujowski and Nagel and Plngot.

For cozpleteness in Table IV we give the recently published results $i c$ : the cross section ratio at the incident photon energy of 1120 ke $\%$. liere the agreement between theory and experinent is also very bad. Studies of the $L$ shell scattering ratios are very linited. :!arty et. al. [78] have published daca for 279 kev photons scattcring from the $L$ shell of thorium $(Z=90)$. Ne can see socie trends if we compare $d a_{K} / d \sigma_{F}$ to $d \sigma_{L} / d \sigma_{F}$ at the same atonic charge and incident photon energy. For small angles $d \sigma_{L} / d \sigma_{F}>d \sigma_{K} / d \sigma_{F}$ and for $\theta \geq 100^{\circ} \mathrm{d \sigma}_{L} / d \sigma_{F}\left\langle d \sigma_{K} / d \sigma_{F}\right.$. For this energy $\left(\omega_{1} \sim 2.5 F_{K}\right.$ and $\omega_{1} \sim$ $14 E_{L}$, where $E_{K}$ and $E_{L}$ are the binding energies of the $k$ and $L$ shells, respectively) binding effects are expected to be large.

There has been much less recent work on the singly differential cross section of total atoms. llubbell et. al.[39] compare the incoherent scattering factors with avallable measurements and conclude that agrement is within the experinental error bars except for Al at $\omega_{1}=8.05 \mathrm{kel}$ and $\mathrm{Pb}$ at $\omega_{1}=280$ and 662 
?age 13

kel at low monentum transfers where the theoretical values are systematically higher. Tlis contrasts with the $K$ shell

ceasurenents where the neasured values are epstematically higher. 
$\checkmark$ Low energy Compton scattering and

the Resonant Raman offect

Contrary to calculations based upon the of $\bar{A}^{2}$ approxination, whlch predict that the scatterfing cross section goes monatomically to zero as the incident photon energy goes to zero, Sparks[80] observed that when the incldent photon energy is near an atonic absorption edge a resonant enhancesent fn the scattering cross section occurs. This phenomena has since been termed the Resonant Raman scattering. This effect - that the spectrum of the scattered $X$ rays is resonantly enhanced at a final photon energy corresponding to a transition between bound states (for example, $k$ shell photoeffect followed by a L-K transition) - can be explained in a single particle rodel. The inconing photon excites a $\mathrm{K}$ electron to a $P$ like continuus stace, an $L$ shell electron drops into the $K$ role emitting a photon of frequency $\omega_{2}\left(=w_{1}-\left(F_{L}+E_{K}\right)\right)$ thereby leaving a hole in the $\mathrm{L}$ shell.

Cavrila and Tugulea[29], independent of the experiments, had found this ?esonant Ranan feature in their analytic calculation of Compton scattering. Extending the work of Cavrila and co-workers[25-29], they showed that light scattered by the $2 \mathrm{p}$ state of atonic hydrogen displayed the following features: (1) A peak displaced from the exciting frequency by the $2 p$ binding energy. This peak corresponds to the Resonant Ranan peak discovered by Sparks; (2) a low frequency - infrared - d1 vergence; (3) a plateau betwe $n$ these two features which is a result of constructive interference between their matrix elenents,

The resonant effect as well as the constructive interference plateau has since been observed by other workers. [81-85] Mlthough the onset of the infrared divergence has yet to be confirmed at these i'cident photon energies. The experimental spectral densities are in qualitative, but not quantitative, agreesent with Cavrila's calculations. This quantitative discrepency, presumably, 
rețlects the differences between hydrogen and a solid. Bannett[83] has irote beyond Gavrila's work and has evaluated the relavent natrix eleatent in a dipole approxtmation using a single slater deteminant constructed frot the one-electron orbitals of Clewent1. [86] This result when scaled to the maximum of the expeciaental curve yields excellent qualitative agreement with their measured curve for $: i \mathrm{i}$ which is near resonance, but is ruch worse for eleaents off resonance.

Thls resonant effect should not be liniced to a final $L$ shell hole, but the effect should be present for any shell hole $(1,: 1, \therefore$, etc.). The il shell hole has been observed by Kodre and Shafroth. [84] Similar resonances can be observed not just near the $x$ edge, but near other edges. 
Conpton scattering is comionly described uthin the nonrelativistic $\hat{A}^{2}$ single particle frozen core formalism. Investigators have evaluated these $\bar{A}^{-2}$ approximations using Hartree-Fock, relativistic, and correlated wave functions. llowever, the validity of the bastc nonrelativistic $\bar{\Lambda}^{-2}$ approximation has never been cstablished.

$A$ correct nonrelativistle description of Compton scattering in a single particle wodel includes both $\mathrm{n}_{n} \vec{A}^{2}$ and a $\bar{p} \cdot \bar{A}$ tern. This description predicts that the cross section do $/ d w_{2}$ diverges as $\omega_{2} \rightarrow 0$. This effect results from the infrared divergence of quantum electrodynamics, and will be present in any more complete theory. The divergence has been observed experimentally and is more proninant at small scattering angles. Calculations limited to the $h^{-2}$ approxitation do not show the effect. In consequence of this divergence the value obtained for the measured singly differential cross section do / $d \Omega$ is dependent upon the energy resolution of the measuring apparatus. This fact rany account for llubbell's clain that $\bar{h}^{-2}$ based incoherent scatter'ng factors agree within experimental error for large scattering angles, but disnyree at small anjles (where the divergent $\bar{p} \cdot \bar{l}$ contribution to the matrix element remeins dominant for tore of the observed spectrum).

Althour llubbell clains good agreenent for photon scattering from the total aton, the agreenent between theory and experir.ent is wuch worse for the differential cross section ratios $d \sigma_{k} / d \sigma_{F}$ and $d \sigma_{L} / d \sigma_{F}$. The matority of these experinents have heen norforned at high incident photon enercies where relativistic effects mav he imoortant.

for incident nioton onergies thove the $k$ edpe of the scattering plement, a resonant pnhancement of the differential eross sertion has heen ohserved for scattered nhoton enersins resultine from bound - hound transitions. This pffect. whirh has 
been called Resonant Raman scatterfug, is not predicted by the simpler $\bar{A}^{-2}$ tased approximations. Qualitative agreement between experiments and theory have been achieved using a single particle $\vec{p} \cdot \bar{A}$ description of the process. Although a description giving quantftaclve agreement will probably require a many body description of the process.

A single particle relativistic calculation of Compton scattering would probably resolve the descrepency tetween thecry and experiment for the differentlal cross section ratios $\mathrm{do} / \mathrm{K} / \mathrm{do}$ and $d \sigma_{L} / \mathrm{do}_{\mathrm{F}}$. It would also, wost likely, improve the agreement between theory and experiment for the singly differential cross section $d \sigma / d \Omega$ at small scattering angles (where the infrared divergence becomes important). At low photon energies such a calculation would probably give qualitative agreament near the ȟsonant Raman peaks.

But a single particle calculation, whether relativistic or nonrelativistic, cannot be expected to resolve discrepencies due to rany bod. effects, such as; scattering from the conduction band electrons in a metal. Many body effects are most Important near the peak in a Compton spectrum, as in the Resonant Raman regime. 


\section{Roferences}

1. A. Somerfold, Atombau and Spekcrallinion, (Vlevez, Itraunsheriez, 1939), Vol, 2, Ch. 8.

2. R. D. Evans, Encyclopedia of Physics, ed. S. Flugge (Springer-Verlag, Berlin, 1958), Vol. 34, D. 210.

3. J. icennan and it. Gavr1la, Phys. Rev. $\wedge$ 15, 1537 (1977).

4. J. H. llubbel and Wm. J. Ve1gle, liss Tech. note 901 (1975).

5. I. A. Bethe and E. E. Salpeter, quancua :lechanics of one- and two-Filectron Atoris, (Plenum, iew York, 1977), p. 295.

6. A. H. Compton, Phys. Rev. 21, 483 (1223).

7. P. A. Ross ankl P. Rirkpatrick, Phys. Rev. 45, 134 (1934); 45, 773 (1934); 46, 668 (1934).

8. J. K. :1. Du:lond, Rev. llod. Phys. 5, 19 (1233).

9. 0. Klein and Y. Rishina, 2. Physik 52, 85.1 (1929); 1. Tart, 1bid. 62, 545 (1930).

10. J. D. 3 jorken and S. D. Drell, Relativistic Quantum:'echanics, (:czeid-lill, lew York, 1964), See. 7.7.

11. F. il. Coensgen, University of Calffornia Radiation Lab. Rept. UCRI, -2413 (1953).

12. L. K. Kurnosova, L. A. Razorenov, and P. A. Cherenkov, 2h. Eskp. Tcor. Fiz. 30, 690 (1956); JLTP 3, 546 (1956).

13. J. D. Anderson, R. W. kenney, and C. A. IcDonald, Jr., Phys. Rev. 102, 1626 (1956).

14. B. Gittelnan, W. C. Parber, I. Selove, D. Tompkins, and F. Forman, Phys. Rev. 171, 1388 (1958).

15. A. T. Goshaw, T. Clanzaan, L. R. Fortney, H. J. Robertson, and H. D. Walker, Phys. Rev. D 18, 1351 (1978). TR/78./

16. I. 11. Brown and R. E. Feynman. Phys. Re" 35. 231 (1952).

17. T. B. Anders, Nuc. Phys. 87. 721 (1969).

13. K. J. York, Phvs. Rey. A 4. 917 (1971): the coefficient of the $\ln |k|$ terc should be $-\frac{5}{6} \pi^{\prime \prime}$ in la.6.2 of this paver.

19. 3. Ram and P. Y. liang. Phvs. Rev. Letr., 26, 476 (1071): 26. 
1210 (E) (1971).

20. R. Ribberfors. J. Ouant. Spectrose. Radiat. Transfer 16. 699 (1976).

21. T. llacada and Y. Nakamura. Yrog. Theor. Phvs. 60, 1717 (1978).

22. H. Cooper. Alv. Phrs, 20, 453 (1971).

23. M. Cavrtlar. Phrs. Rev. A G, 1348 (1972).

24. 11. A. Yraners and V. Heisenberg, Z. Pivsik 31. 681 (1925):

I. Valler. ibid. 51.213 (1928): 58.75 (1929).

25. H. K. Tseng. II. Gavrilia. and R. II, Rratt, :PS Rent. nn. 2 (1973) (unpublished).

26. I1. Givrila. Phus. Rev. A 6. 1360 (1972).

27. \%. Gavrila. Rev. Ronk. Plivs. 19. 473 (1974).

28. A. Costescu and 1 . Cavrila. Rev. Roum. Pins. 18. 493 (1773).

29. I. Cavrila and I. i.. Tugulea, Rov. Roun. Phvs. 20, 209 (1975).

30. See. for example. J. H. Jauch and F. Mohrlich. The Theory of Photons and l'lectrons. (Addison-Weslev. Cambridge . lass.. $1955)$.

31. P. Eisenzereer and P. H. Platzman. Phvs. Rev. A 2, 415 (1970).

32. F. Schrisite. Ann. Pinsik 21. 89 (1934).

33. Z. Sulkowski and B. inee1, Arkiv. Fusik 20. 323 (1961).

34. S. Shimizu. Y. Lakavama, and T. llukovama, Phvs. Rev. 140. A806 (1965).

35. I. P. llanson. F. llernan. J. D. Tea, and S. Skillman, Acta Cryst. 17. 1040 (1964).

36. D. T. Croner. J. Chem. Phys. 50. 4857 (1969): Los Alamos Scientific Lab. Redt. LA4079 (1959).

37. E. Storn and II. I. Israel, Nuc. Data Tables A7, 565 (1970).

38. Vin. Je Veigle. Atomic Data 5. 51 (1973).

39. J. H. Ilubbell. Wn. J. Veigle, E. A. Brigas. R. T. Brown,

D. T. Cromer, and B. J. llowerton. J. Chek. Phys. Ref. Data 4, 471 (1975).

40. G. E. Killby, Proc. Phys. Soc. 86, 1037 (1965).

41. L. B. Iendelsohn. and V. il. Smith. Jr.. Compton Scattering. ed. 3. Hillans (HcGiaw !ill, Kew York. 1977), Ch. 5. 
42. 8. IIIlans. see other chaters of llef. 41.

43. :1. Codper. Contemp. Phys, 18, 489 (1977).

44. F. Briegs. L. B. Hendelsohn, and J. B. Blann, Atomic Data and Ruclear Data Tables 16. 201 (1975).

45. F. Briges. L. B. Mendelsohn, and J. B. Mann. Sandia Lab. Rept. 75-0636 (1976).

46. v. 11. Srith. Jr. and R. E. Brown. Chem. Phvs. Tett. 20, $42 \pi$ (1973).

47. II. liaon and 1\%. Coraille, J. Phys. B 6. 954 (1973).

48. T. Paakkari. li, F. Bergeren, R. Ribberfors, and V. !!alnen. Phvs. Rev. B 14. 2301 (1976).

49. B. J. Block and L. B. !!endelsohn. Phys. Rev. $\Lambda$ 9. 129 (1074).

50. J. Randles, Proc. Phys. Soc. A70, 337 (1957).

51. K. C. Standing and J. V. Jovanovich. Can. J. Phvs, 40. 622 (1962).

52. ii. Schumacher. Z. Pliysik 242. 444 (1971).

53. 11. Pracoux. 11. Meunier, It. Avon, and G. Roche, Phvs. Rev. A 16. 2022 (1977).

54. 1. :. Furry. Phys, Rev. 81, 115 (1951).

55. One can derive the nonrelativistic result Eo. (7) bv startine fron the exact relativistic matrix elenent for a bound electron and taking the nonrelativistic. limit ignoriag soins. see A. I. Nkhlezer and V. B. Berestetskif. Quantun Electrodvnamics. (Interscience, New York, 1965), Sec. 35.1.

56. G. E. Irown, R. E. Peirerls, and J. B. loodward, 2roc. R. Soc. A 227, 51 (1954).

57. H. R. Johnson and F. D. Felock, Phys. Rev. 168, 22 (1969).

58. L. Kissel and R. Il. Pratt, Pinys, Rev. Lett, 40,387 (1978).

59. E. Henry. (Phd. thesis, unpublished, 1969).

60. 1. B. Whittingham, J. Phys. A 4, 21 (1971).

61. L. Uittwer, (Phd thesis, unpublished, 1972).

62. D. A. Owen, Phys. Rev. A 16, 1594 (1977); Phys. Lett. 69A, 177 (1978).

63. G. C. Spitale, Lawrence t,ivermore Lab. Rept. LC?L-51596 
(1974).

64. P. ... Baba Prasad, C. Basuaraju and P. P. Kane, Phys. Rev. A 15, 1934 (1977).

65. P. Rulliusen and :1. Schunacher, J. Phys. B 9, 2435 (1976).

66. D. Brint, E. Fuschini, l. T. Crinellini, and D. S. R. :furty, iusovo Ciriento 16, 727 (1960).

67. J. H. Hotz and C. Hissoni, Phys. Rev. 124, 1458 (1961).

69. J. Varna and :1. A. Eswaram, Phys. Rev. 127, 1197 (1962).

69. L. V. East and E. R. Lewis, Physica 44, 595 (1969).

68. A. Ramalinga Reddy, V. Lakshminarayana, and S. Jnanananda, Ind. J. Pure Appl. Phys. 4, 371 (1966).

71. 0. Pingot, Juc. Phys. 1119, 667 (1963); J. de Physique 32, $413(1971)$.

72. 5. I.. Chintalapudi and k. Parthasaradhi Ind. J. Phys. 43, 492 (1969).

73. D. S. R. :!urty, D. v. Kirishna Reddy, and E. Karasimhacharyulu, Ind. J. Zure Appi. Phys. 9, 305 (1970); Physica 75, 394 (1974).

74. A. Karaisisa Reddy, V. Latshminarayana, and S. Jnanananda, Proc. Tis. Soc. 91, 71 (1967).

75. 0. Pingot, Nuc. Phys. A133, 334 (1969); J. de Physique 33, 189 (1972).

76. D. S. R. Hurty, V. Govindareddy, and E. Narasimhacharyulu, J. Phys. A $\underline{6}, 265$ (1973).

77. D. S. ?. Wurty, V. Covinda Reddy, and C. Kageswara Rao, Physica 92C, 137 (1977); J. Pnys. 3 10, 47 (1977).

78. S. T. P. V. J. Swazy and D. S. R. :!urey, Physica 84C, 289 (1976).

79. P. I. Baba Prasad and P. P. Kane, J. of Research NBS 78A, 461 (1974); Phys. Rev. A 15, 1976 (1977).

80. C. J. Sparks, Jr., Phys. Rev. Lett. 33, 262 (1974).

81. Y. B. Bannett, J. I. Cersten, 1.. Tzoar, and I. Freund, Phys. Rev, Lett. 36, 882 (1976).

82. P. Eisenberger, P. I. Platzman, and H. Winick, 
Phys, Rev. Latt. J6, 623 (1976); Phys. Rev. $8 \underline{13}, 2377$ (1276).

83. Y. B. Bannett, D. C. Rapaport, and I. Freund, phys. Sev. A 16 , 2011 (1977).

84. A. F. Kodre and S. M. Shafroth, Phys. Rev. $\Lambda 19,675(1979)$.

85. J. H. Hall, K. A. Jamison, O. L. Weaver, and P. Richardson, Phys. Rev. A 19, 568 (1970).

86. E. Clementi, IBM J. Res. 9, 2 (1965). 
Figure Captions

Fig. 1. Locus of equal atomic cross sections for Compton and photoeffect interactions $(\sigma=t)$, and for Compton and patr-production interactions $(\theta=K)$. The incident photon energy is $h v$, and $z$ is the atonic number of the atons in the absorber. Compton collisions have larger cross sections than any other mode of interaction in the entire dojain of medium energy photons marked "Compton effect dominant". (from Ref. 2).

Fig. 2 Cosparison of the püre Coulomb $k$ shell Compton scattering cross section $d^{2} d d \Omega d_{2}$ for $z=82, w_{1}=662 \mathrm{keV}$ and $\theta=0$ between the relativistic results of Whittingham (nef. 60), of the $\bar{A}^{2}$ approximation of Schnaidt (Ref. 32), of the inpulse approxination Eq. (24), and of the $\bar{A}^{-2}$ togetler with the dipole $\bar{p} \cdot \bar{A}$ formula of Gavrila. The binding energ $E_{K}$ is calculated nonrelativistically. The Conpton eneraj is indicated by a straight 11ne. (fron Ref. 25).

Fig. 3. Sata as Fig. 1 except $\theta=60^{\circ}$.

Fig. 4. Sace as Fig. 1 except $\theta=180^{\circ}$.

Fig. 5. Comparison of the pure Coulomb $k$ shell scattering cross section $d \sigma / d \Omega$ for $z=82, \psi_{1}=662(\mathrm{keV})$ between, the relativistic results of Whittinghan and of various other approxinations. Symbols $3 P$ and $R$ refer to nonrelativistic and relativistic cases. (from Ref, 25) 
TABLE I. Experimental crass section ratios $d \sigma_{k} / d \sigma_{F}$ for an incident photon energy of $279 \mathrm{keV}$. The reference from which the values were obtained is given next to the chemfeal bymbol.

\begin{tabular}{|c|c|c|c|c|c|c|}
\hline$\theta^{\circ}$ & $\begin{array}{c}A_{L} \\
\text { (Ref. } 75)\end{array}$ & $\begin{array}{c}\text { Sm } \\
\text { (Ref. 75) }\end{array}$ & $\begin{array}{c}\text { Ta } \\
\text { (Ref. 75) }\end{array}$ & $\begin{array}{c}\text { W } \\
\text { (Ref. 76) }\end{array}$ & $\begin{array}{c}\text { Au } \\
\text { (Ref. 75) }\end{array}$ & $\begin{array}{c}\text { Tlı } \\
\text { (Ref. 77) } \\
\end{array}$ \\
\hline 20 & $.158 \pm .03$ & $.161 \pm .06$ & $.189=.04$ & & $.206 \pm .02$ & \\
\hline 30 & & & & $.267 \pm .04$ & & $.330 \pm .04$ \\
\hline 50 & & & & $.456 \pm .06$ & & $.391 \pm .015$ \\
\hline 55 & $.542 \pm .04$ & $.294 \pm .08$ & $.290 \pm .04$ & & $.317 \pm .03$ & \\
\hline 70 & & & & $.689 \pm .04$ & : & $.571 \pm .04$ \\
\hline 90 & $.844 \pm .08$ & $.550 \pm .08$ & $.358 \pm .06$ & & $.378 \pm .03$ & \\
\hline 105 & & & & $.819 \pm .04$ & & $.751 \pm .05$ \\
\hline 125 & $1.039 \pm .06$ & $.691 \pm .06$ & $.487 \pm .05$ & $.94 \pm .08$ & $.426 \pm .03$ & $.908 \pm .17$ \\
\hline 150 & & & & & & $1.160 \pm .04$ \\
\hline 160 & $1.076 \pm .07$ & $.740 \pm .04$ & $.502 \pm .07$ & & $.449 \pm \ldots .02$ & \\
\hline
\end{tabular}


TABLE II. Experimental crosg section ratios do ${ }_{K} / d \sigma_{F}$ for an Incident photon energy of 320 keV. otherwlse the same as Table I.

\begin{tabular}{|c|c|c|c|c|c|c|}
\hline $0^{\circ}$ & $\begin{array}{c}\sin \\
\text { (Ref. 74) } \\
\end{array}$ & $\begin{array}{c}\mathrm{Sm} \\
\text { (Ref. 72) }\end{array}$ & $\begin{array}{c}\text { Ta } \\
\text { (Ref, 74) } \\
\end{array}$ & $\begin{array}{c}\text { Ta } \\
\text { (Ref. 72) }\end{array}$ & $\begin{array}{c}\mathrm{Pb} \\
\text { (Ref. 74) }\end{array}$ & $\begin{array}{c}\mathrm{Pb} \\
\text { (Ref. 72) }\end{array}$ \\
\hline 45 & $.645 \pm .097$ & $.65 \pm .10$ & $.525 \pm .079$ & $.53 \pm .08$ & $.50 \pm .075$ & $.50 \pm .08$ \\
\hline 60 & $.750 \pm .113$ & $.75 \pm .11$ & $.660 \pm .099$ & $.66 \pm .1$ & $.595 \pm .089$ & $.60 \pm .1$ \\
\hline 90 & $.825 \pm .124$ & $.83 \pm .13$ & $.745 \pm .112$ & $.75 \pm .11$ & $.700 \pm .105$ & $.70 \pm .11$ \\
\hline 110 & $.905 \pm .136$ & $.91 \pm .14$ & $.850 \pm .128$ & $.85 \pm .13$ & $.835 \pm .125$ & $.84 \pm .13$ \\
\hline
\end{tabular}


TABLE III. Experimental cross gection ratios for an Ircident photon energy of 662 keV. Otherwlse the same as Table I.

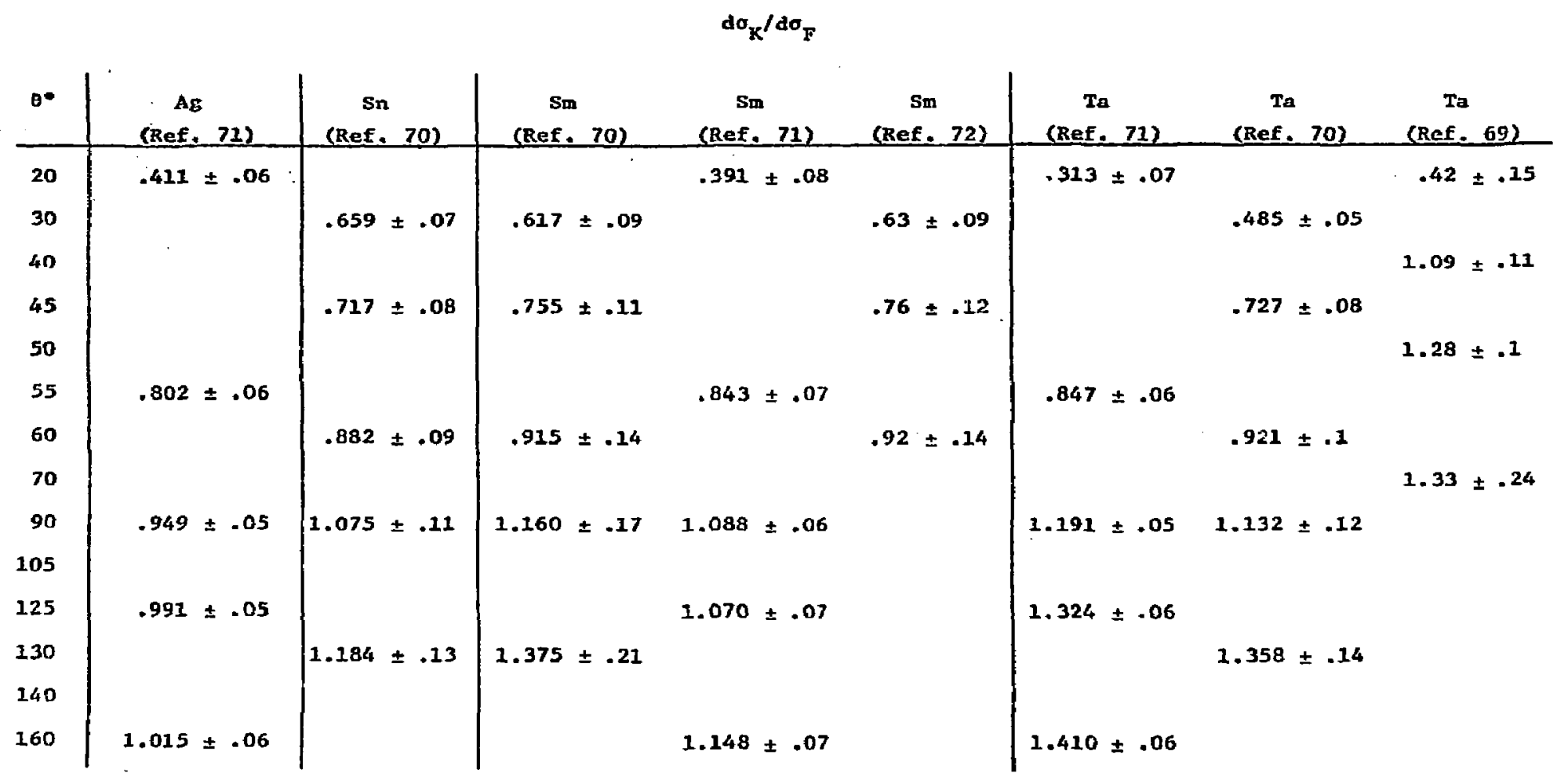




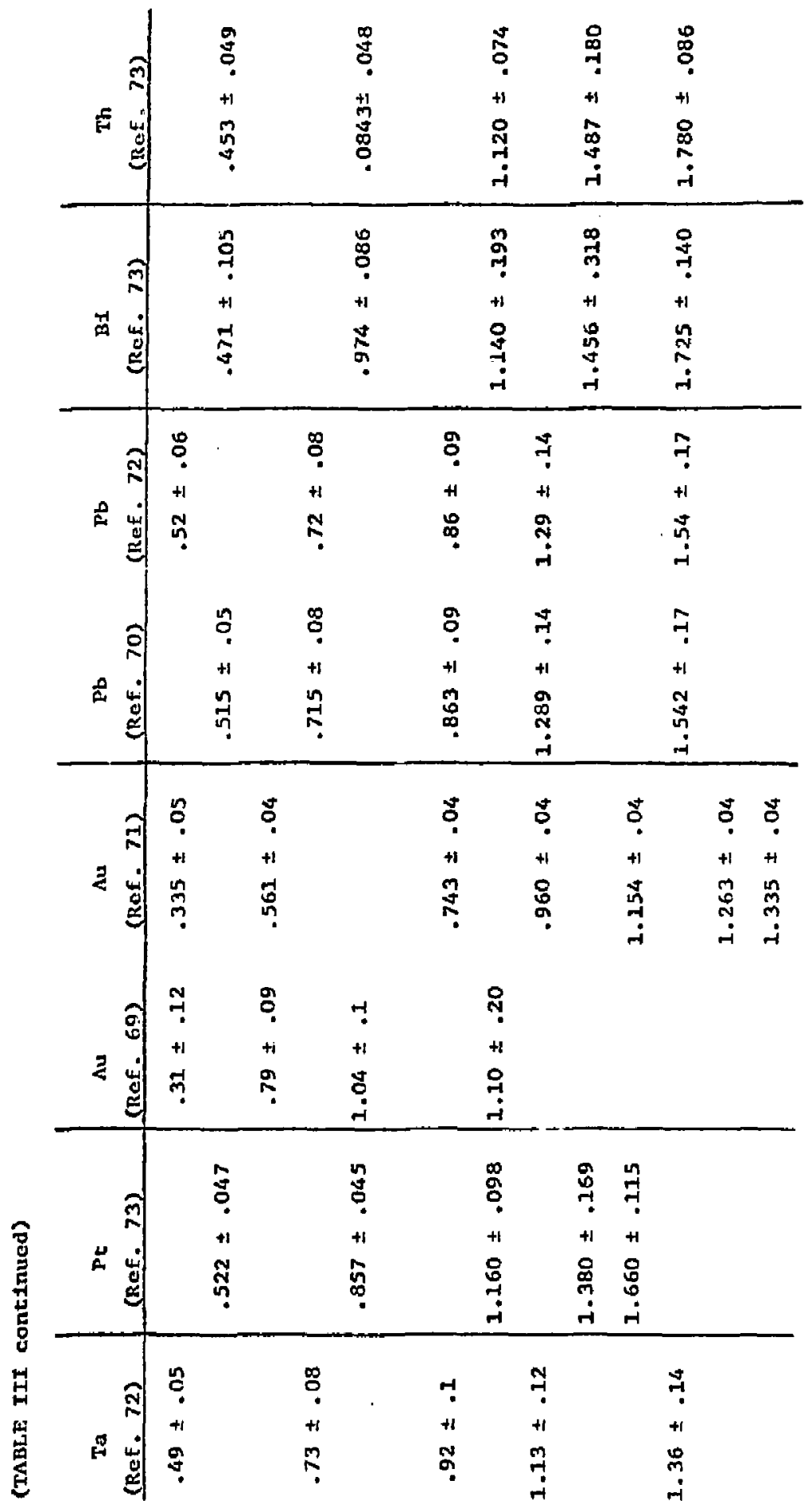


TABLE IV. Experimental cross section ratios for an incident photon energy of $1120 \mathrm{kev}$. Otherwise the same as Table $I$.

\begin{tabular}{|c|c|c|c|c|c|}
\hline$\theta$ & $\begin{array}{c}\text { sn } \\
\text { (Ref. 79) }\end{array}$ & $\begin{array}{c}\text { Ta } \\
\text { (Ref. 79) }\end{array}$ & $\begin{array}{c}d \sigma_{K} / d \sigma_{F} \\
\text { Au } \\
\text { (Ref. 79) }\end{array}$ & $\begin{array}{c}\text { Pb } \\
\text { (Ref. 79) }\end{array}$ & $\begin{array}{c}\text { Th } \\
\text { (Ref. 79) } \\
\end{array}$ \\
\hline 25 & $.71 \pm .07$ & & $.63 \pm .09$ & & $.40 \pm .13$ \\
\hline 60 & $.86 \pm .08$ & & $.81 \pm .09$ & $.86 \pm .1$ & $.91 \pm .11$ \\
\hline 90 & $.94 \pm .08$ & & $1.33 \pm .12$ & & $1.36 \pm .12$ \\
\hline 100 & & & $1.44 \pm .15$ & & $1.20 \pm .39$ \\
\hline 120 & & $.91 \pm .26$ & $.98 \pm .15$ & & $.93 \pm .15$ \\
\hline
\end{tabular}




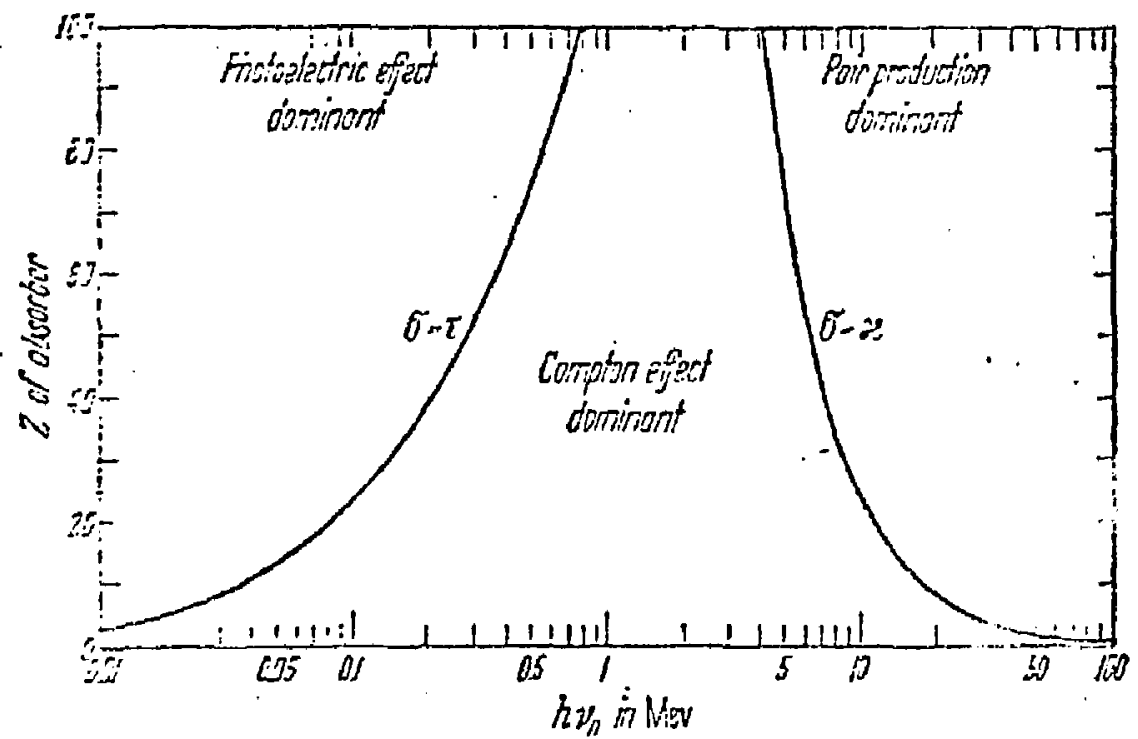

Fig. 1 


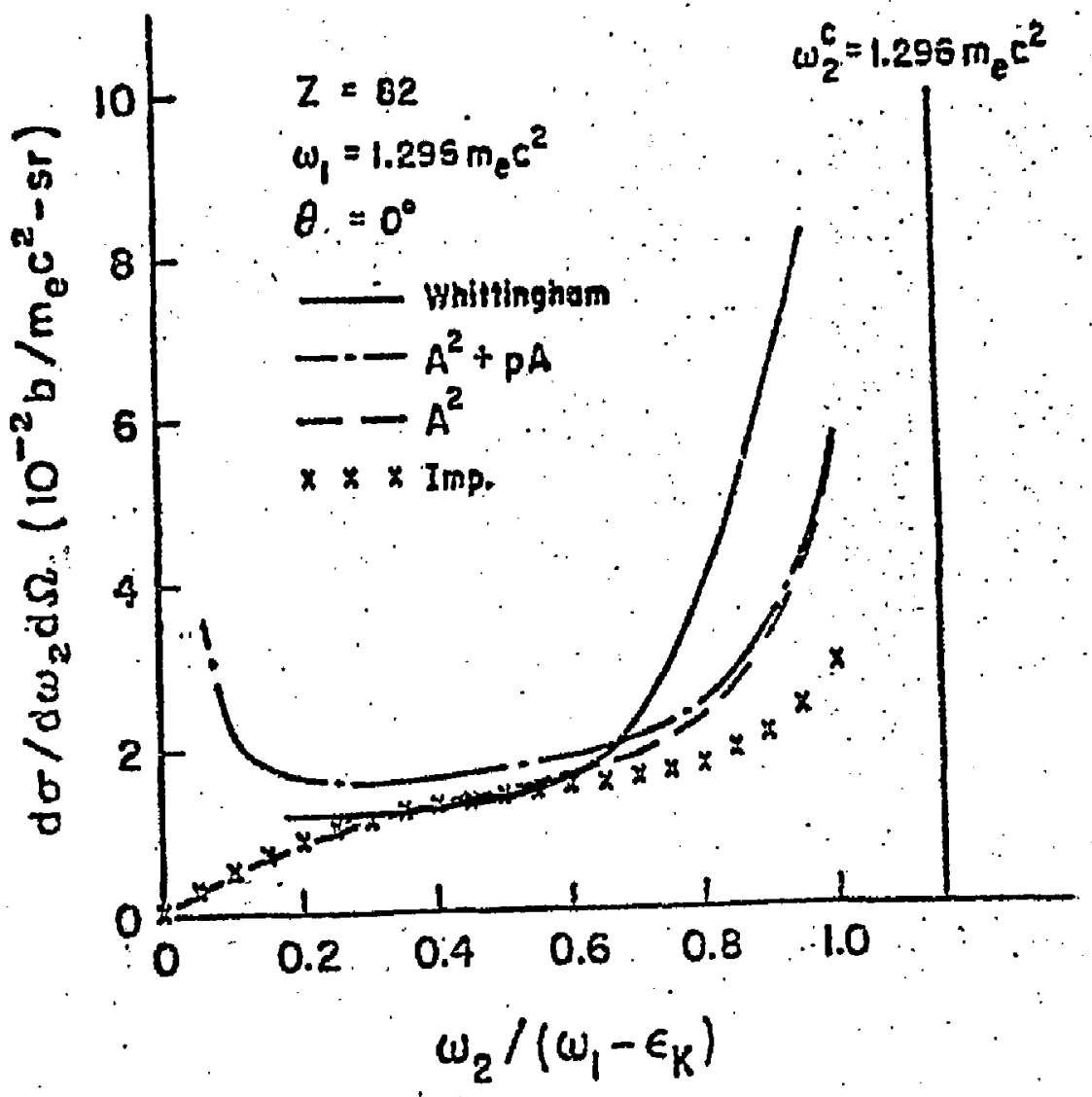

Figure 논 


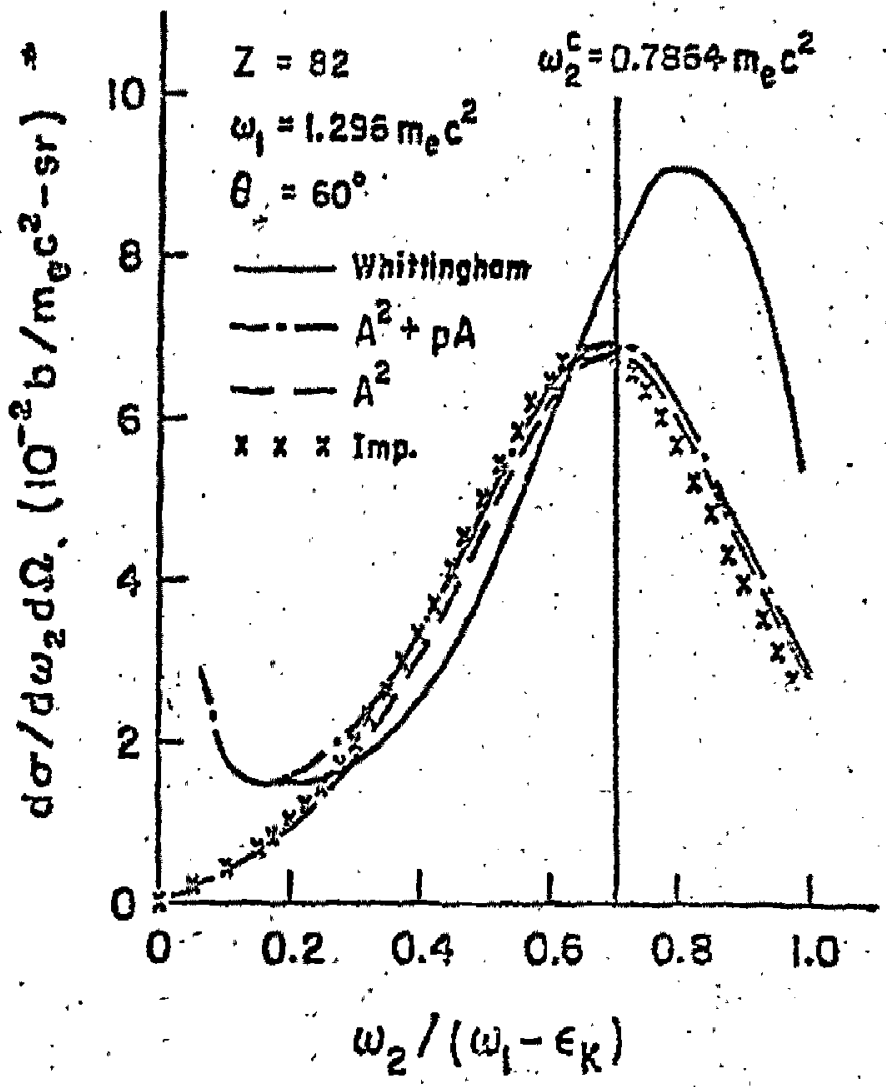

Pigure $\frac{z}{3}$ 


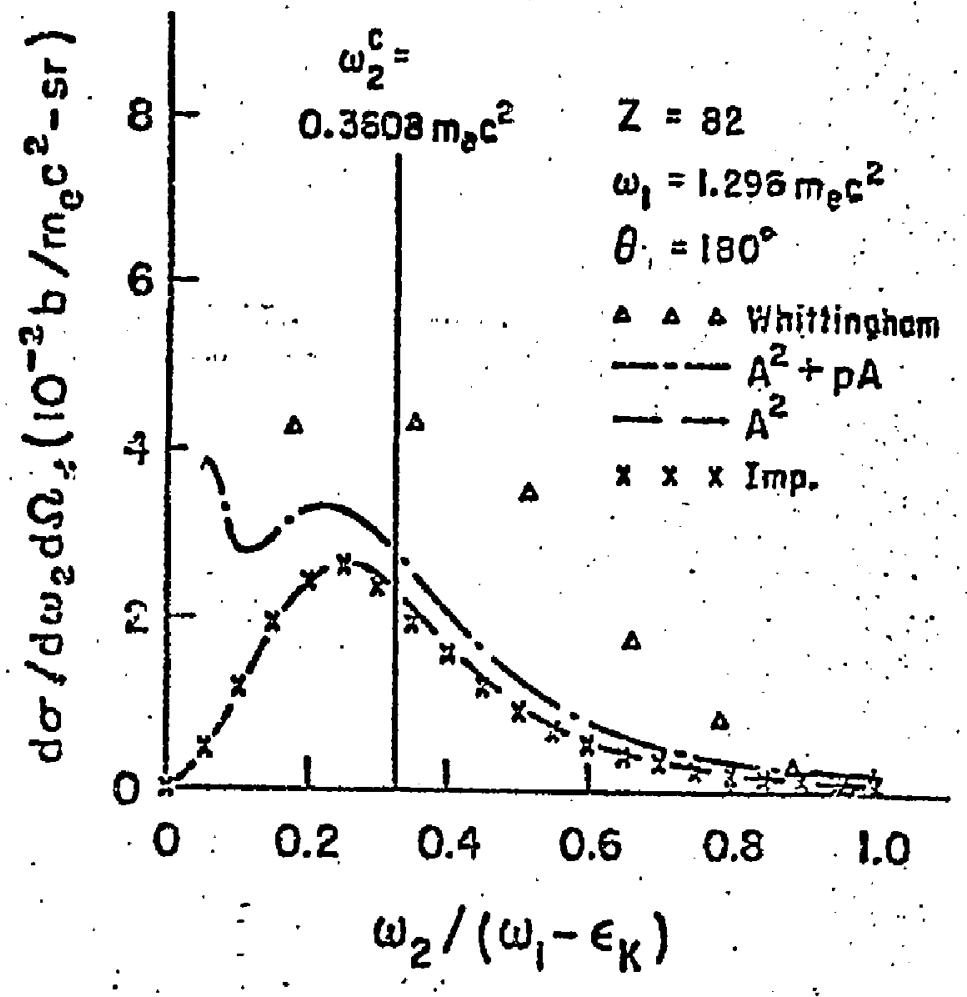

Figure $\frac{\xi}{\rho}$ 


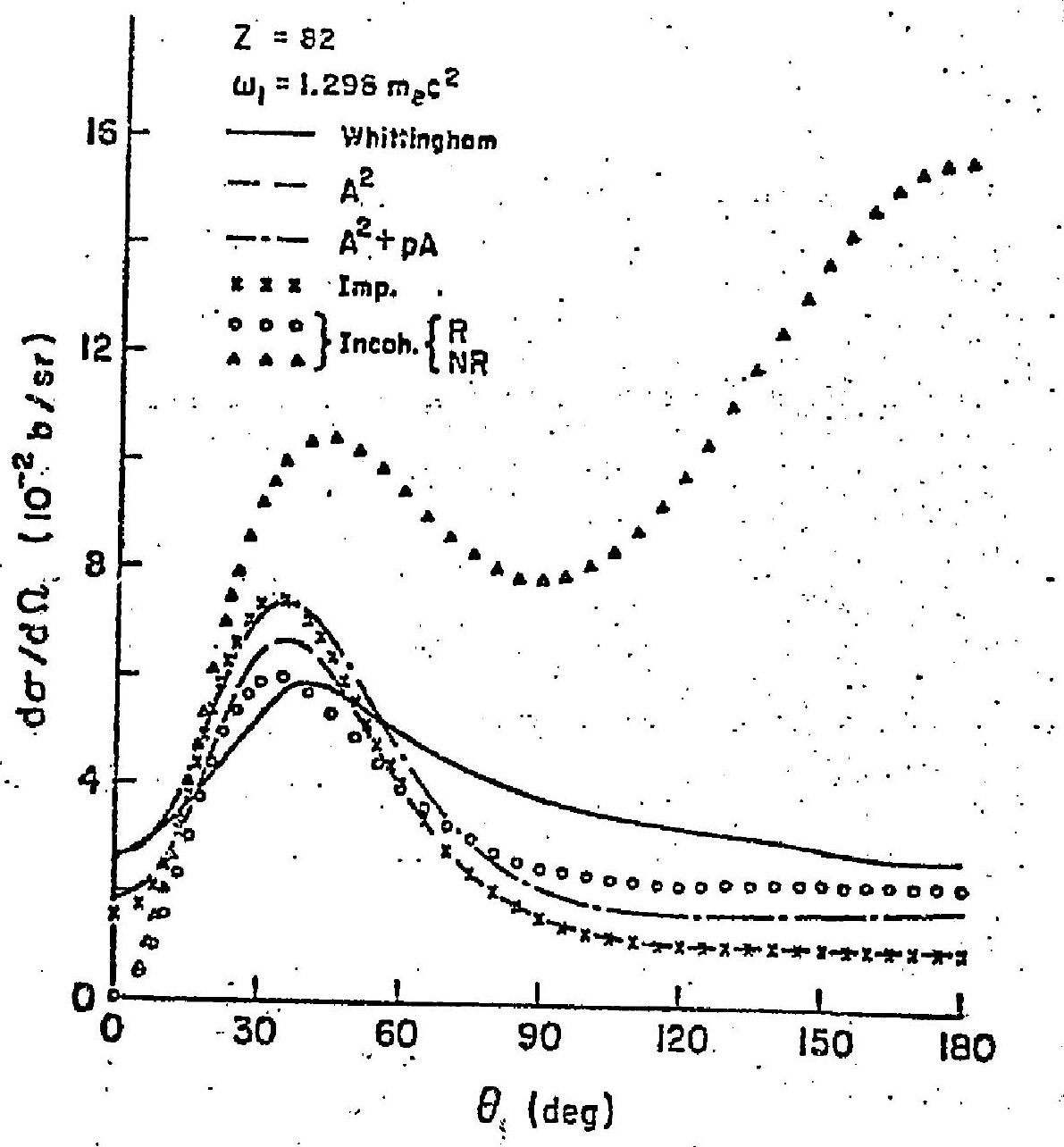

Figure 5 